\title{
Gravitation Astrometric Measurement Experiment (GAME)
}

\author{
Mario Gai ${ }^{1}$, Alberto Vecchiato ${ }^{1}$, Alessandro Sozzetti ${ }^{1}$, \\ Sebastiano Ligori ${ }^{1}$ and Mario G. Lattanzi ${ }^{1}$ \\ ${ }^{1}$ Istituto Nazionale di Astrofisica - Osservatorio Astronomico di Torino, \\ V. Osservatorio 20, I-10025 Pino T.se (TO), Italy \\ email: gai@oato.inaf.it
}

\begin{abstract}
GAME (Gravitation Astrometric Measurement Experiment) is a mission concept based on astronomical techniques for high precision measurements of interest to Fundamental Physics and cosmology, in particular the $\gamma$ and $\beta$ parameters of the Parameterized PostNewtonian formulation of gravitation theories extending the General Relativity.

High precision astrometry also provides the light deflection induced by the quadrupole moment of Jupiter and Saturn, and, by high precision determination of the orbits of Mercury and high elongation asteroids, the PPN parameter $\beta$.

The astrometric and photometric capabilities of GAME may also provide crucial complementary information on a selected set of known exo-planets.
\end{abstract}

Keywords. gravitation, astrometry, techniques: miscellaneous, space vehicles: instruments

\section{Introduction: the main science goal of GAME}

The main goal of GAME (Gravitation Astrometric Measurement Experiment) is to estimate the $\gamma$ and $\beta$ parameters of the Parameterized Post-Newtonian (PPN) formalism, by repeated measurement from space of the star light deflection close to the solar limb. The mission is conceived as a novel rendition, from space and using modern technology, of the experiment by Dyson, Eddington and collaborators conducted during the solar eclipse of 1919, when the gravitational bending of light was measured for the first time. This makes GAME a decisive experiment for the understanding of gravity physics, cosmology and the Universe evolution at a fundamental level.

General Relativity can act as a cosmological attractor for scalar-tensor theories, with expected deviations in the $10^{-5}-10^{-8}$ range for $\gamma$, as from Damour \& Nordtvedt (1993). Also, the experimental evidence of an accelerated expansion of the Universe is interpreted as a long range perturbation of the gravity field of the visible matter, generated by Dark Energy, in addition to the measurements, at different scale length, explained with nonbarionic Dark Matter (e.g. galaxy rotation curves). An alternative explanation might be provided by a modified version of General Relativity, e.g. in which the curvature invariant $R$ is no longer constant as in Einsteins equations, i.e. the $f(R)$ gravity theories. $\mathrm{A}<10^{-7}$ level determination of $\gamma$ will provide stringent constraints on acceptable theories, as derived e.g. in Capozziello \& Troisi (2005).

The original experiment of Dyson, Eddington and collaborators confirmed the General theory of Relativity at $10^{-1}$-level. The same technique was used for several decades after 1919, but its accuracy was not significantly improved, because of fundamental experimental limitations: the short time available for the observations (limited by the eclipse duration, which constrained also the number and brightness of the observed stellar sample), the atmospheric disturbances, and the background from the solar corona. The best 


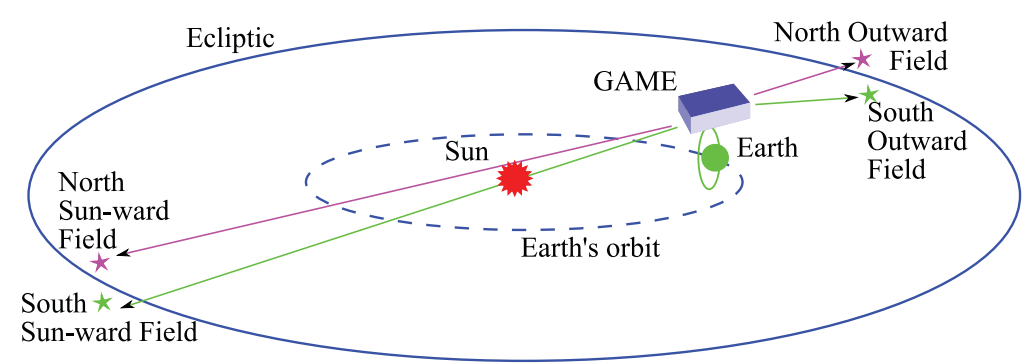

Figure 1. The GAME satellite, observing field pairs either close to, or far away from, the Sun.

current estimate of $\gamma$ was achieved from the Cassini data, reaching the $10^{-5}$ level of accuracy, by Bertotti et al. (2003), exploiting the derivative of the Shapiro effect.

In the near future, and focusing on astrometric measurements, the most promising effort presently under development is the Gaia mission, which, building on the same principles of Hipparcos, might achieve a level of accuracy of $10^{-6}-10^{-7}$ during the second half of the next decade, as shown in Vecchiato et al. (2003).

GAME, described in a small mission version in Gai et al. (2009), merges astrometric and coronagraphic techniques to perform micro-arcsec differential determination of the deflection angle on stellar field pairs, measured in two epochs: close to the Sun and in opposition, after six months (Fig. 1). GAME requires a limited time to fulfil its main science goal, since the $\gamma$ determination is most conveniently performed on the high stellar density regions of intersection between the Ecliptic plane and the Milky Way disc.

\section{Exo-planetary system applications of GAME}

GAME will carry out a robust, adaptive additional science program using the available fraction of observing time. Its high-precision, fully differential astrometry, and its highaccuracy, high-cadence photometry, make it an ideal instrument for a program targeted to well-selected, known extrasolar planetary systems, critically deepening our understanding of key issues in exo-planet astrophysics. The goal is four-fold: 1) improving the orbital phase coverage against bad and/or insufficient sampling; 2) looking for planetary companions at all orbital periods; 3) looking for low- as well as high-mass components (Super Earths, Neptunes, and Jupiters); 4) refining the characterization of multiple planet systems (including accurate coplanarity measurements).

The three-fold exo-planet science program is focused on: a) GAME astrometry of nearby, bright stars known to host one or more exoplanets through long-term highprecision RV monitoring; b) GAME follow-up studies of Gaia-detected exoplanets around stars of varied masses, metallicities, and ages (with and without ground-based RV support); c) GAME astro-photometric investigations of planetary systems in which one or more component is known to transit.

\section{References}

Damour, T. \& Nordtvedt, K. 1993, Phys. Rev. Lett. 70, 2217

Capozziello, S. \& Troisi, A. 2005, Phys. Rev. D 72, 044022

Bertotti, B., Iess, L., \& Tortora, P. 2003, Nature 425, 374

Vecchiato, A., Lattanzi, M. G., Bucciarelli, B., Crosta, M., de Felice, F., \& Gai, M. 2003, A\&A 399,337

Gai, M., Vecchiato, A., Ligori, S., Fineschi, S., \& Lattanzi, M. G. 2009, Proc. SPIE 7438, 74380 T 